that melanophore-expanding hormone may possess some slight inherent adrenocorticotrophic activity. B. G. BENFEY

Department of Pharmacology, McGill University, Montreal.

M. SAFFRAN

A. V. Sohatly

Allan Memorial Institute of Psychiatry, McGill University, Montreal.

${ }^{1}$ Saffran, M., and Bayliss, M. J., Endocrinol., 52, 140 (1953). Saffran, M., and Schally, A. V. (in preparation).4

2 Stehle, R. I., J. Pharmacol. Exp. Therap., 57, 1 (1936); Rev. Can. Biol., 3, 408 (1944). Addendum in Benfey, B. G., Brit. J. Pharmacol. 8, 435 (1953).

\section{Salicyloylhydrazide : a Reagent of Wide Use in Organic and Histochemical Analysis}

Present methods of demonstrating ketosteroids in tissues are generally regarded as being unsatisfactory. 2-Hydroxy-3-naphthoyl hydrazide ${ }^{1}$ is the reagent most recently introduced for this purpose, yet it condenses only sluggishly with carbonyl groups and fails to distinguish between aldehydes and ketones. A number of phenolic hydrazides have since been tested. Of these, $2: 4$ dihydroxybenzoylhydrazide ${ }^{2}$ is better in that it provides azo-hydrazones of different colours in the ketosteroid group ${ }^{3}$, but it still lacks adequate condensing reactivity.

Salicyloylhydrazide ${ }^{4}$ is at present under investigation, and the preliminary results now reported may prove of interest to workers both in histochemistry and in other fields. It has been found that the aldehydic salicyloylhydrazones fluoresce brilliantly in diverse colours under ultra-violet light, whereas the ketonic derivatives which have been studied exhibit a uniform dull blue fluorescence. Zinc acetate greatly intensifies the ketonic fluorescence, but has no appreciable effect on the fluorescence of the aldehydic derivatives. A similar but very much weaker reaction is obtained with cadmium salts. Neither the reagent nor the carbonyl compound separately shows any luminescence at low $p \mathrm{H}$.

The colour and intensity of fluorescence of the salicyloylhydrazones may be further controlled in two fundamental ways : (1) chemically, by alteration of $p \mathrm{H}$, by treatment with free halogens, and by chelation; and (2) optically, by using an instrument capable of offering a series of monochromatic beams of ultra-violet light of differing wave-lengths. Preliminary experiments, utilizing varying combinations of these two control procedures, indicate that it may be possible to devise a system capable of distinguish ing individual components of a mixture of ketones.

Investigations have also been carried out to determine quantitatively the condensing reactivity of the reagent. In a typical experiment, dehydroepiandrosterone was condensed with salicyloylhydrazide in acetic acid solution and excess reagent removed by washing with dilute hydrochloric acid. The salicyloylhydrazone, in virtue of its being a phenol, was then converted to an indoaniline by coupling it with $p$-aminodiethylaniline (stabilized with sulphur dioxide), in the presence of periodic acid as oxidant. The intensely coloured blue-green indoaniline was extracted with ethyl acetate and the optical density read in an absorptiometer against a blank determination, using a suitable filter. A linear relation was found between the optical density and the amount of ketosteroid present over the range $5-50 \mu \mathrm{gm}$.
Quantitative estimation of ketosteroids by fluorimetry of the salicyloylhydrazones is being studied in order to achieve an analytical method of a more direct and simple character.

Although salicyloylhydrazide has been investigated in the first instance as a reagent for use in histochemistry, the indoaniline reaction for phenols and also the fluorescent reaction of zinc acetate with salicyloylhydrazones are obviously capable of much wider application.

In general, salicyloylhydrazide is superior to the previous hydrazides I have described ${ }^{1,2}$ in respect of reactivity in coridensation, in differentiating capacity and in lending itself to colorimetric determination of ketosteroids.

The detailed use of the foregoing procedures in histochemistry and in relation to the estimation of ketosteroids will be the subject of future communications.

I am grateful to Prof. J. Patterson for his guidance throughout and to Prof. R. J. Harrison and Dr. G. H. Bourne for histochemical facilities provided at the London Hospital Medical School. The optical experiments were carried out at the National Institute for Medical Research in conjunction with $\mathrm{Mr}$. J. Smiles, to whom I am indebted for the special knowledge and advice he contributed.

Charing Cross Hospital,

B. Camber London, W.C.2.

Nov. 3.

${ }^{1}$ Camber, B., Nature, 163, 285 (1949).

'Camber, B., and Dziewiatkowski, D. D., J. Amer. Chem. Soc., 73, 4021 (1951).

${ }^{3}$ Camber, 3 . (unpublished results).

Curtius, Th., Struve, A., and Radenhausen, R., J. prakt. Chem., 52, $227(1895)$.

\section{Lingual Proprioception in Pig, Dog and Cat}

THe muscle-spindles, shown by Cooper ${ }^{1}$ to exist in the intrinsic tongue muscles of man and rhesus monkey, have not so far been satisfactorily demonstrated in other species.

Cooper has failed to find proprioceptive end-organs in the intrinsic musculature of the tongue in lamb and kitten, and this finding has been confirmed and extended by a histological survey of mammalian. tongues in the course of this work.

However, the pattern of innervation in the tongues of pig, dog and cat suggests that proprioceptive impulses in these animals may be, at least in part, conveyed through a certain specialized form of receptor found in the intramuscular connective tissue, which is sufficiently constant in all three species to justify further attention.

Thick frozen sections, prepared by the diammine-silver ion method of Garven and Gairns ${ }^{2}$, show that these end-organs are formed by a coil of axis-cylinder enclosed in an oval or pear-shaped capsule, which appears to be a terminal dilation of the neurilemma. The long axis of the capsule lies parallel to that of the muscle fibres. The endings occur mainly in the intrinsic museles and are most abundant between the vertical and transverse groups, where they are closely associated with the strata of motor innervation in this region. A few endings were found in the inferior longitudinal muscle; none was noted in the superior longitudinal.

The structure and position of the intramuscular endings suggest that deformation of the capsule 